\title{
REMARKS ON A PAPER BY W. A. DAY ON A MAXIMUM PRINCIPLE UNDER NONLOCAL BOUNDARY CONDITIONS*
}

\author{
BY \\ BERNHARD KAWOHL \\ Universität Heidelberg
}

\begin{abstract}
In a recent paper W. A. Day proved a decay property for solutions to a linear parabolic equation with nonlocal boundary conditions. Such boundary conditions arise in thermoelasticity. We extend his result (a) from one to arbitrary space dimensions, (b) from linear to nonlinear parabolic equations, and (c) from differential equations to differential inequalities. Our tool is the classical maximum principle.
\end{abstract}

Let $\Omega \subset \mathbf{R}^{n}(n \geqslant 1)$ be a bounded domain with smooth boundary $\partial \Omega$, e.g., with boundary of class $C^{1}$. Let $u: \bar{\Omega} \times \mathbf{R}^{+} \rightarrow \mathbf{R}$ be a classical solution to the differential inequality

$$
\frac{\partial u}{\partial t}(x, t)-A u(x, t)+c(x) f(u(x, t)) \leqslant 0 \quad \text { in } \Omega \times \mathbf{R}^{+}
$$

under the nonlocal boundary condition

$$
u(x, t)=\int_{\Omega} u(y, t) g(x, y) d y \quad \text { on } \partial \Omega \times \mathbf{R}^{+} .
$$

Here $A$ is an operator

$$
A u=\sum_{i, j=1}^{n} a_{i j}(x, u, D u) \frac{\partial^{2} u}{\partial x_{i} \partial x_{j}}+\sum_{i=1}^{n} b_{i}(x, u, D u) \frac{\partial u}{\partial x_{i}},
$$

which satisfies the ellipticity assumption

$$
a|p|^{2} \leqslant \sum_{i, j=1}^{n} a_{i j}(x, q, p) p_{i} p_{j}
$$

for some uniform $a>0$, every $x \in \Omega, q \in \mathbf{R}$, and $p \in \mathbf{R}^{n}$, and the symbol $D u$ denotes the spatial gradient of $u$. The function $f: \mathbf{R} \rightarrow \mathbf{R}$ is monotone nondecreasing and satisfies $f(0)=0$ by assumption. Finally, the coefficients $a_{i j}, b_{i}$, and $c$ as well as $f$ are assumed to

\footnotetext{
* Received July 26, 1985.
} 
be sufficiently regular, e.g., continuous in their arguments, and $c(x)$ is supposed to be nonnegative.

The weight function $g: \bar{\Omega} \times \bar{\Omega} \rightarrow \mathbf{R}$ in boundary condition (2) is also assumed continuous. Boundary conditions of this type arise in the theory of thermoelasticity. We refer to the paper of W. A. Day for the physical background and to [1,3] for related problems.

Under the above assumptions we shall prove the following

THEOREM 1 . Let $u \geqslant 0$ be a classical solution to problem (1), (2) and suppose

$$
\int_{\Omega}|g(x, y)| d y<1 \text {. }
$$

Then the function $h(t):=\sup _{x \in \Omega} u(x, t)$ is a nonincreasing function of $t$ for $t>0$. An immediate consequence is formulated as

COROllary 2. Let $u$ be a classical solution of the differential equation

$$
\frac{\partial u}{\partial t}-A u+c f(u)=0 \quad \text { in } \Omega \times \mathbf{R}^{+}
$$

under boundary condition (2) and suppose that (3) holds. Then $e(t):=\sup _{x \in \Omega}|u(x, t)|$ is a nonincreasing function of $t$ for $t>0$.

Let us now prove the theorem under the additional assumption that strict inequality holds in (1). The extension of the proof to weak inequality is then standard; for instance, see the classical book of Protter and Weinberger [4]. If $h$ is not nonincreasing then there exists a positive time $T$ and a point $x \in \bar{\Omega}$ such that a positive maximum of $u$ over $\bar{\Omega} \times[0, T]$ is attained in $x, T$. We distinguish two cases. If $x \in \Omega$, then $(\partial u / \partial t)(x, T) \geqslant 0$ and $D u(x, T)=0$. Furthermore, the Hessian matrix of $u$ is negative semidefinite, so that the strict version of (1) cannot hold. If $x \in \partial \Omega$ then condition (2), the maximality of $u$ in $x$, and (3) yield the contradiction

$$
u(x, T)=\int_{\Omega} g(x, y) u(y, T) d y \leqslant u(x, T) \int_{\Omega}|g(x, y)| d y<u(x, T) .
$$

The proof of the corollary follows from looking at the function $v=u^{2}$.

Acknowledgment. I am gratefully indebted to P. Bader for some comments which led to an improvement of a first draft of this paper.

\section{REFERENCES}

[1] P. Bader, On a quasilinear elliptic boundary value problem of nonlocal type with an application in combustion theory, Z. Angew. Math. Phys. 35, 771-779 (1984)

[2] W. A. Day, A decreasing property of solutions of parabolic equations with applications to thermoelasticity, Quart. Appl. Math. 40, 468-475 (1983)

[3] B. P. Paneyakh, Certain nonlocal boundary value problems for linear differential operators, Math. Notes Acad. Sci. USSR 35, 223-229 (1984)

[4] M. H. Protter and H. F. Weinberger, Maximum principles in differential equations, Prentice-Hall, Englewood Cliffs, N. J., 1967 